\title{
Beatty sequences and multiplicative number theory
}

\author{
by
}

\author{
A. G. Abercrombie (Liverpool)
}

Notation and conventions. If $X$ is any set we denote the characteristic function of $X$ by $\chi_{X}$.

The set of positive integers or natural numbers is denoted by $\mathbb{N}$, the set of non-negative integers by $\mathbb{N}_{0}$. If $x$ is a real number the symbols $\lfloor x\rfloor$, $\lceil x\rceil$ denote respectively the greatest integer $\leq x$ and the least integer $\geq x$. The fractional part $x-\lfloor x\rfloor$ of $x$ is denoted by $\langle x\rangle$ and the toral norm $\min (x-\lfloor x\rfloor,\lceil x\rceil-x)$ by $|x|_{\mathbb{T}}$. The residue class of an integer $h$ modulo a fixed prime will be denoted by $\bar{h}$.

The symbol $\gamma$ denotes Euler's constant.

The Fourier transform of a 1-periodic function $f$ will be denoted by $\widehat{f}$.

If $f$ and $g$ are real functions we use the notation $f \ll g$ to mean the same as $f=O(g)$. If $\lim _{x \rightarrow \infty} f(x) / g(x)=1$ we write $f \sim g$.

1. Introduction. Let $\theta$ be any number in $[1, \infty)$. The sequence $B_{\theta}=$ $\{\lfloor\theta n\rfloor: n \in \mathbb{N}\}$ is called the Beatty sequence determined by $\theta$. Beatty sequences have been the subject of intensive investigation in recent years on account of their connection with semigroups (see for example [8] and the references therein); however this connection does not appear to be relevant to the question we consider in the present paper. A classical result in prime number theory (see [2], Theorem 9.9) states that for each irrational $\theta$ the sequence $B_{\theta}$ contains infinitely many primes. Equivalently this result states that for each irrational $\theta$ we have

$$
\liminf _{n \rightarrow \infty} \tau(\lfloor\theta n\rfloor)=2,
$$

where as usual $\tau(n)$ denotes the number of divisors of $n$. In the present paper we consider the average behaviour of $\tau(\lfloor\theta n\rfloor)$. This does not appear to have been investigated before.

We write

$$
T(\theta ; x)=\sum_{n \leq x / \theta} \tau(\lfloor\theta n\rfloor)=\sum_{n \leq x, n \in B_{\theta}} \tau(n) .
$$


We shall see that the behaviour of $T(\theta ; x)$ is related to the behaviour of sums of the form

$$
T(\lambda, x)=\sum_{n \leq x} \tau(n) e^{2 \pi i n \lambda}
$$

which have been studied by S. Chowla [1] and others. Making use of this relationship we shall prove the following:

TheOREM I. For all irrational $\theta>1$ we have

$$
T(\theta ; x) \sim \theta^{-1} T(1 ; x) .
$$

TheOREM II. For almost all $\theta \geq 1$ with respect to Lebesgue measure and for each $\varepsilon>0$ we have

$$
T(\theta ; x)=\theta^{-1} T(1 ; x)+O\left(x^{5 / 7+\varepsilon}\right),
$$

where the $O$-constant may depend on $\theta$ and on $\varepsilon$.

The term $T(1 ; x)$ appearing on the right in Theorems I and II is just the well-known sum $\sum_{n \leq x} \tau(n)$. An account of some of the work done on the evaluation of this sum may be found in [3]. However, only the elementary estimate

$$
T(1 ; x)=x \log x+(2 \gamma-1) x+O\left(x^{1 / 2}\right)
$$

is relevant in the present context since the more refined estimates introduce main terms that are absorbed in our error term. The author does not know whether or to what extent the exponent $5 / 7$ in the statement of Theorem II can be improved.

It is easy to see that Theorem I becomes false if the hypothesis that $\theta \notin \mathbb{Q}$ is dropped: in particular it follows from Lemma 2.3 below that $T(p ; x) \sim$ $p^{-1} T(1 ; x)$ does not hold for any prime $p$. The reader might wonder however whether a version of Theorem II, possibly with a poorer error term, might hold with "all irrational $\theta$ " in place of "almost all $\theta$ ". In fact we shall see that this is not the case. More precisely we shall prove the following:

TheOREM III. Let $g: \mathbb{R} \rightarrow \mathbb{R}$ be positive, increasing and unbounded. Then for uncountably many numbers $\theta>1$ there exist arbitrarily large positive $x$ such that the relation

$$
\left|T(\theta ; x)-\theta^{-1} T(1 ; x)\right| \leq T(1 ; x) / g(x)
$$

does not hold.

2. Some lemmata. In this section we collect together some lemmata required for the proofs of our main results.

We begin with estimates involving the divisor function $\tau$.

Lemma $2.1([6]$, Theorem $6 \cdot 5 \cdot 2)$. For each $\varepsilon>0$ we have

$$
\tau(n)=O\left(n^{\varepsilon}\right) .
$$


Lemma 2.2 ([1], Theorem 5). For each irrational $\lambda$ we have

$$
T(\lambda, x)=o(T(1 ; x)) .
$$

For integers $h, k$ we write

$$
T(h, k ; x)=\sum_{n \leq x, n \equiv h(\bmod k)} \tau(n) .
$$

The following lemma is a special case of a formula given by D. R. HeathBrown in [4] but implicit in earlier work of Ramanujan ([9], p. 82).

LEMma 2.3. For each prime $p$ we have

$$
T(h, p ; x) \sim \begin{cases}\frac{2 p-1}{p^{2}} T(1 ; x) & \text { if } p \mid h, \\ \frac{p-1}{p^{2}} T(1 ; x) & \text { otherwise. }\end{cases}
$$

We shall require some results from Diophantine approximation theory. Let $\theta>0$ be an irrational number and let $\left(p_{n} / q_{n}\right)_{n \in \mathbb{N}_{0}}$ be the sequence of best rational approximations to $\theta$, that is, we take $p_{0}$ to be the nearest integer to $\theta$ and $q_{0}=1$, while for $n \in \mathbb{N}$ we choose $p_{n}, q_{n} \in \mathbb{N}$ minimising $q_{n}$ subject to the condition

$$
\left|\theta-\frac{p_{n}}{q_{n}}\right|<\left|\theta-\frac{p_{n+1}}{q_{n+1}}\right| .
$$

For any increasing function $g: \mathbb{R} \rightarrow \mathbb{R}$ satisfying $g(1)=1$ we say that $\theta$ is of principal cotype $g$ if given any $B \geq 1$ we have $B \leq q_{n} \leq B g(B)$ for some $n$. The following lemma is a simple consequence of Khintchine's theorem on metric Diophantine approximation ([7], Ch. II, Theorem 4).

Lemma 2.4. Given $\varepsilon>0$, for almost all $\theta$ with respect to Lebesgue measure there exists a function $g=g_{\theta}: \mathbb{R} \rightarrow \mathbb{R}$ such that

$$
g(x)=O\left(\log ^{1+\varepsilon} x\right)
$$

and such that $\theta$ is of principal cotype $g$.

The next result is taken from [7], p. 42. The reader will observe that our definition of principal cotype is slightly more restrictive than the definition in [7]: in Lang's notation, we are assuming $g\left(B_{0}\right)=B_{0}=1$. The statement of the following lemma has been modified accordingly.

Lemma 2.5. For any irrational $\theta$ of principal cotype $g$ we have

$$
\sum_{1 \leq n \leq x}|\csc \pi n \theta| \ll x \log x+x g(x) .
$$

Here the implied constant does not depend on $\theta$. 
Given an irrational number $\theta$ and a positive number $x$ the discrepancy $D(\theta ; x)$ is defined by setting

$$
D(\theta ; x)=\sup _{0 \leq a<b \leq 1}\left|b-a-x^{-1} \sum_{1 \leq n \leq x} \chi_{[a, b)}\langle n \theta\rangle\right| .
$$

The following lemma is taken from [5], p. 96.

Lemma 2.6. Let $\theta$ be irrational, let $\left(p_{n} / q_{n}\right)_{n \in \mathbb{N}_{0}}$ be the sequence of best rational approximations to $\theta$, and for $x \geq 1$ let $h(x)=h_{\theta}(x)$ be the greatest integer satisfying $q_{h(x)} \leq x$. Then for all $x \geq 1$ we have

$$
D(\theta ; x) \leq 8 x^{-1} \sum_{0 \leq n \leq h(x)} \frac{q_{n+1}}{q_{n}} .
$$

It is well known (see for example [7], Ch. I) that for any irrational $\theta$ the numbers $q_{n}$ satisfy $q_{n+2} \geq q_{n+1}+q_{n}$. Therefore the function $h_{\theta}$ defined in the statement of Lemma 2.6 satisfies $h_{\theta}(x) \ll \log x$. Thus using Lemma 2.6 we obtain the following:

COROLlary 2.7. For any irrational $\theta$ of principal cotype $g$ we have

$$
D(\theta ; x) \ll x^{-1} g(x) \log x .
$$

Let $M$ be a natural number. For each integer $m$ write $\widetilde{m}=m /(M+1)$ and for $m \neq 0$ set

$$
a_{m}=-\frac{\pi \widetilde{m}(1-|\widetilde{m}|) \cot (\pi \widetilde{m})+|\widetilde{m}|}{2 \pi i m} .
$$

J. D. Vaaler in [10] (see also [3], p. 111) found that the trigonometric polynomials

$$
\psi_{M}(t)=\sum_{1 \leq|m| \leq M} a_{m} e^{2 \pi i m t}
$$

are exceptionally good approximations to the "sawtooth" function $\psi$ given by $\psi(t)=\langle t\rangle-1 / 2$. To be precise we have the following (see [3], Theorem A6):

LEMmA 2.8. For each natural number $M$ we have

$$
\left|\psi_{M}(t)-\psi(t)\right| \leq(2 M+2)^{-1} \sum_{0 \leq|m| \leq M}(1-\widetilde{m}) e^{2 \pi i m t} .
$$

We end this section by deriving from Lemma 2.8 a more convenient bound for the "error" $\left|\psi_{M}(t)-\psi(t)\right|$.

COROLlary 2.9. For each natural number $M$ we have

$$
\left|\psi_{M}(t)-\psi(t)\right| \leq \frac{1}{2}(M+1)^{-2}|\csc (\pi t)|^{2} .
$$


Pr o of. Using Lemma 2.8 we have

$$
\begin{aligned}
\left|\psi_{M}(t)-\psi(t)\right| & \leq \frac{1}{2}(M+1)^{-1} \sum_{|m| \leq M}(1-|\widetilde{m}|) e^{2 \pi i m t} \\
& =\frac{1}{2}(M+1)^{-2} \sum_{|m| \leq M}(M+1-|m|) e^{2 \pi i m t} \\
& =\frac{1}{2}(M+1)^{-2} \sum_{0 \leq M^{\prime} \leq M} \sum_{-M^{\prime} \leq m \leq M^{\prime}} e^{2 \pi i m t} \\
& =\frac{1}{2}(M+1)^{-2} \sum_{0 \leq M^{\prime} \leq M} \frac{e^{2 \pi i\left(M^{\prime}+1\right) t}-e^{-2 \pi i M^{\prime} t}}{1-e^{2 \pi i t}} \\
& \leq 2(M+1)^{-2}\left|1-e^{2 \pi i t}\right|^{-2}=\frac{1}{2}(M+1)^{-2}|\csc (\pi t)|^{2}
\end{aligned}
$$

as required.

3. Proofs of Theorems I and II. For each $\theta \geq 1$ we shall denote by $\xi_{\theta}$ the characteristic function of the set

$$
\left\{t \in \mathbb{R}: 1-\theta^{-1} \leq\langle t\rangle<1\right\} .
$$

Our proofs of Theorems I and II both start from the easily verified identity

$$
T(\theta ; x)=\sum_{n \leq x} \tau(n) \xi_{\theta}(n / \theta) .
$$

Since $\xi_{\theta}$ is 1-periodic we can use Fourier methods to approximate the right hand side of (3.1) by a linear combination of the sums

$$
T(m / \theta, x)=\sum_{n \leq x} \tau(n) e^{2 \pi i m n / \theta}
$$

Estimating these sums by Lemma 2.2 yields Theorem I directly but a more delicate analysis is needed to obtain Theorem II.

Proof of Theorem I. Let $\theta$ be a fixed irrational number in $(1, \infty)$ and write $\xi=\xi_{\theta}$. For a given $\varepsilon>0$ let $\xi^{-}, \xi^{+}$be continuously differentiable 1-periodic functions satisfying

$$
0 \leq \xi^{-} \leq \xi \leq \xi^{+}
$$

and

$$
\theta^{-1}-\varepsilon / 3 \leq \int_{0}^{1} \xi^{-}, \quad \int_{0}^{1} \xi^{+} \leq \theta^{-1}+\varepsilon / 3 .
$$

The Fourier series $\widehat{\xi}^{-}(n), \widehat{\xi}^{+}(n)$ are absolutely convergent, so we may choose 
$M=M(\varepsilon)$ satisfying

$$
\max \left(\sum_{|m|>M}\left|\widehat{\xi}^{-}(m)\right|, \sum_{|m|>M}\left|\widehat{\xi}^{+}(m)\right|\right) \leq \varepsilon / 3 .
$$

By Lemma 2.2 we may choose $X \in \mathbb{R}$ so large that $x>X$ implies

$$
\begin{aligned}
& \sum_{n \leq x} \tau(n) \sum_{1 \leq|m| \leq M} \widehat{\xi}^{-}(m) e^{2 \pi i m n / \theta} \leq \frac{\varepsilon}{3} T(1 ; x), \\
& \sum_{n \leq x} \tau(n) \sum_{1 \leq|m| \leq M} \widehat{\xi}^{+}(m) e^{2 \pi i m n / \theta} \leq \frac{\varepsilon}{3} T(1 ; x) .
\end{aligned}
$$

From (3.2) we obtain

$$
\theta^{-1}-\varepsilon / 3 \leq \widehat{\xi}^{-}(0), \quad \widehat{\xi}^{+}(0) \leq \theta^{-1}+\varepsilon / 3 .
$$

Hence we find using (3.3), (3.4) and (3.5) that

$$
\begin{aligned}
T(\theta ; x) & =\sum_{n \leq x} \tau(n) \xi(n / \theta) \geq \sum_{n \leq x} \tau(n) \xi^{-}(n / \theta) \\
& =\sum_{n \leq x} \tau(n)\left(\widehat{\xi}^{-}(0)+\left(\sum_{1 \leq|m| \leq M}+\sum_{|m|>M}\right) \widehat{\xi}^{-}(m) e^{2 \pi i m n / \theta}\right) \\
& \geq\left(\theta^{-1}-\varepsilon\right) T(1 ; x)
\end{aligned}
$$

for all $x>X$ and similarly

$$
T(\theta ; x) \leq\left(\theta^{-1}+\varepsilon\right) T(1 ; x) .
$$

Since $\varepsilon>0$ was arbitrary the theorem is proved.

Proof of Theorem II. Fix $\varepsilon>0$ and let $g: \mathbb{R} \rightarrow \mathbb{R}$ be an increasing function satisfying

$$
g(1)=1, \quad g(x)=O\left(\log ^{1+\varepsilon} x\right) .
$$

Let $\theta>1$ be a fixed irrational number such that $\theta^{-1}$ is of principal cotype $g$. We will show that (1.1) holds for such a $\theta$ : Theorem II is an immediate consequence of this fact in conjunction with Lemma 2.4.

We write $\xi=\xi_{\theta}$ and observe that for $t$ outside the countable set $\mathbb{Z} \cup$ $\left(\mathbb{Z}-\theta^{-1}\right)$ we have

$$
\xi(t)=\theta^{-1}+\psi(t)-\psi\left(t+\theta^{-1}\right) .
$$

Since $\theta$ is irrational we have $n / \theta \in \mathbb{Z} \cup\left(\mathbb{Z}-\theta^{-1}\right)$ only when $n=0$ or $n=-1$, so using (3.6) in (3.1) we obtain

$$
\begin{aligned}
T(\theta ; x) & =\theta^{-1} T(1 ; x)+\sum_{n \leq x} \tau(n)\left(\psi\left(\frac{n}{\theta}\right)-\psi\left(\frac{n+1}{\theta}\right)\right) \\
& =\theta^{-1} T(1 ; x)+R(\theta ; x),
\end{aligned}
$$


say. We note that for fixed $M \in \mathbb{N}$ (to be determined later) we have

$$
\begin{aligned}
|R(\theta ; x)| \leq & \left|\sum_{n \leq x} \tau(n) \psi_{M}\left(\frac{n}{\theta}\right)\right|+\left|\sum_{n \leq x} \tau(n) \psi_{M}\left(\frac{n+1}{\theta}\right)\right| \\
& +\sum_{n \leq x} \tau(n) R_{M}(n) \\
= & \left|S_{0}\right|+\left|S_{1}\right|+S_{2},
\end{aligned}
$$

say, where we define

$$
R_{M}(n)=\left|\psi_{M}\left(\frac{n}{\theta}\right)-\psi\left(\frac{n}{\theta}\right)\right|+\left|\psi_{M}\left(\frac{n+1}{\theta}\right)-\psi\left(\frac{n+1}{\theta}\right)\right| .
$$

We establish bounds for $\left|S_{0}\right|$ and $\left|S_{1}\right|$ as follows. Using the easily verified inequality

$$
|x(1-x) \cot (\pi x)| \leq 1
$$

(valid for $0 \leq x \leq 1$ ), we see that the coefficients $a_{m}$ of $\psi_{M}$ satisfy $\left|a_{m}\right| \leq$ $|m|^{-1}$. We thus have

$$
\begin{aligned}
\left|S_{j}\right| & =\left|\sum_{n \leq x} \tau(n) \psi_{M}\left(\frac{n+j}{\theta}\right)\right| \\
& =\left|\sum_{|m| \leq M} a_{m} e^{2 \pi i j m} \sum_{n \leq x} \tau(n) e^{2 \pi i m n / \theta}\right| \\
& \leq 2 \sum_{1 \leq m \leq M} m^{-1}|T(m / \theta, x)| .
\end{aligned}
$$

Now (cf. [1], p. 552) we have

$$
\begin{aligned}
T(m / \theta, x) & =2 \sum_{l \leq(x / m)^{1 / 2}} \sum_{l \leq n \leq x / l} e^{2 \pi i l m n / \theta}+O\left(x^{1 / 2} m^{1 / 2}\right) \\
& =O\left(\sum_{l \leq(x / m)^{1 / 2}}|\csc (\pi l m / \theta)|\right)+O\left(x^{1 / 2} m^{1 / 2}\right) .
\end{aligned}
$$

Since $\theta^{-1}$ is of principal cotype $g$, Lemma 2.5 implies

$$
\begin{aligned}
\sum_{l \leq(x / m)^{1 / 2}}|\csc (\pi l m / \theta)| & \leq \sum_{l \leq x^{1 / 2} m^{1 / 2}}|\csc (\pi l / \theta)| \\
& \ll x^{1 / 2} m^{1 / 2}\left(\log \left(x^{1 / 2} m^{1 / 2}\right)+g\left(x^{1 / 2} m^{1 / 2}\right)\right) \\
& \ll\left(x^{1 / 2} m^{1 / 2}\right)^{1+\varepsilon}
\end{aligned}
$$

for any $\varepsilon>0$. Combining (3.9), (3.10) and (3.11) we obtain

$$
S_{j} \ll\left(x^{1 / 2} M^{1 / 2}\right)^{1+\varepsilon}
$$

for $j=0$ or 1 . 
We now establish a bound for $S_{2}$. By Corollary 2.9 we have

$$
\begin{aligned}
S_{2} & \ll M^{-2} \sum_{n \leq x} \tau(n)\left(|\csc (\pi n / \theta)|^{2}+|\csc (\pi(n+1) / \theta)|^{2}\right) \\
& \ll M^{-2} \sum_{n \leq x} \tau(n)\left(\left|\frac{n}{\theta}\right|_{\mathbb{T}}^{-2}+\left|\frac{n+1}{\theta}\right|_{\mathbb{T}}^{-2}\right),
\end{aligned}
$$

where ||$_{\mathbb{T}}$ denotes toral norm. We partition the natural numbers $\leq x$ into disjoint sets $A$ and $B$ in the following way: we put $n \in A$ if

$$
\min \left(\left|\frac{n}{\theta}\right|_{\mathbb{T}},\left|\frac{n+1}{\theta}\right|_{\mathbb{T}}\right) \leq M^{-2 / 3}
$$

and $n \in B$ otherwise. Then since trivially $\left|\psi_{M}-\psi\right| \leq 1$ we have

$$
S_{2} \ll \sum_{n \in A} \tau(n)+M^{-2 / 3} \sum_{n \in B} \tau(n) .
$$

By Corollary 2.7 we have

$$
\left|\#(A)-2 x M^{-2 / 3}\right| \ll \log ^{2+\varepsilon} x
$$

so that

$$
\#(A) \ll x M^{-2 / 3} .
$$

Using Lemma 2.1 we find that

$$
\sum_{n \in A} \tau(n) \ll x^{1+\varepsilon} M^{-2 / 3}
$$

(for any $\varepsilon>0$ ), while

$$
\sum_{n \in B} \tau(n) \leq T(1 ; x) \leq x^{1+\varepsilon}
$$

Substituting in (3.13) we have

$$
S_{2} \ll x^{1+\varepsilon} M^{-2 / 3} .
$$

Combining (3.8), (3.12) and (3.14), and choosing $M=\left\lceil x^{3 / 7}\right\rceil$ we obtain

$$
R(\theta ; x) \ll x^{5 / 7+\varepsilon}
$$

and using this in (3.7) we see that (1.1) holds for $\theta$ as claimed. As we mentioned previously, this fact together with Lemma 2.4 yields the Theorem.

4. Proof of Theorem III. We have already remarked that if $\theta$ is rational then in general we do not have $T(\theta ; x) \sim \theta^{-1} T(1 ; x)$. Suppose now that $\theta=\theta_{1}+\theta^{\prime}$ where $\theta_{1}=p / q$ is rational and $\theta^{\prime}>0$ is small. Then we have $\lfloor\theta n\rfloor=\left\lfloor\theta_{1} n\right\rfloor$ for $n \leq x$, say, where $x$ is comparable with $q / \theta^{\prime}$. This implies $T(\theta ; x)=T\left(\theta^{\prime} ; x\right)$. Given a function $g$ satisfying the hypothesis of 
Theorem III and a large positive $x$, we find that if $\theta=\theta_{1}+\theta^{\prime}$ for suitable rational $\theta_{1}$ and small enough $\theta^{\prime}>0$ we can ensure

$$
\left|T(\theta ; x)-\theta^{-1} T(1 ; x)\right|>T(1 ; x) / g(x) .
$$

We will prove Theorem III by showing that there are uncountably many $\theta$ each having arbitrarily close rational approximations of this kind. Our method in this section is vaguely reminiscent - especially as regards the appeal to Dirichlet's theorem on primes in progressions - of Chowla's method in [1], Section 15.

Proof of Theorem III. For an arbitrary real function $G$ we will call a sequence $p_{1}<p_{2}<\ldots$ of primes $G$-good if for each natural number $n$ we have

$$
\begin{gathered}
p_{n+1} \equiv 1\left(\bmod p_{n}\right), \\
\frac{p_{n+1}}{p_{n+2}-1} \leq\left(\frac{p_{n}}{p_{n+1}-1}\right)^{2}
\end{gathered}
$$

and

$$
p_{n+1} \geq 1+G\left(p_{n}\right) .
$$

Dirichlet's theorem on primes in arithmetic progressions implies that there are uncountably many $G$-good sequences (for fixed $G$ ).

For any sequence $\mathcal{P}=\left(p_{j}\right)_{j \in \mathbb{N}}$ of primes and for each $j \geq 1$ write

$$
p_{j}^{\prime}=\frac{p_{j}}{p_{j+1}-1} .
$$

We set $P_{0}=1$ and for $n \geq 1$ we set $P_{n}=\prod_{j \leq n} p_{j}^{\prime}$. Also if $\mathcal{P}$ satisfies (4.2) we can clearly define a real number $\theta_{\mathcal{P}}$ by setting

$$
\theta_{\mathcal{P}}=p_{1}+\sum_{n \in \mathbb{N}} P_{n}
$$

We claim that for $G$-good sequences $\mathcal{P}$ the map $\mathcal{P} \mapsto \theta_{\mathcal{P}}$ is injective: it follows that there exist uncountably many real numbers $\theta>1$ such that $\theta=\theta_{\mathcal{P}}$ for some $G$-good $\mathcal{P}$.

Thus let $\mathcal{P}=\left(p_{j}\right), \mathcal{Q}=\left(q_{j}\right)$ be distinct sequences of primes satisfying (4.1) and (4.2), and define $\left(q_{j}^{\prime}\right),\left(Q_{j}\right)$ analogously with $\left(p_{j}^{\prime}\right),\left(P_{j}^{\prime}\right)$ : we must show that $\theta_{\mathcal{P}} \neq \theta_{\mathcal{Q}}$. If $p_{1} \neq q_{1}$ one checks easily that

$$
\left\lfloor\theta_{\mathcal{P}}\right\rfloor=p_{1} \neq q_{1}=\left\lfloor\theta_{\mathcal{Q}}\right\rfloor
$$

so that certainly $\theta_{\mathcal{P}} \neq \theta_{\mathcal{Q}}$ in this case. If $p_{1}=q_{1}$ let $m>1$ be the least integer such that $p_{m}^{\prime} \neq q_{m}^{\prime}$ and suppose without loss of generality that $p_{m}^{\prime}<q_{m}^{\prime}$. We will show that $\theta_{\mathcal{P}}<\theta_{\mathcal{Q}}$. We have clearly

$$
\theta_{\mathcal{Q}}-\theta_{\mathcal{P}}=\sum_{n \geq m}\left(Q_{n}-P_{n}\right)
$$


Now since $p_{m}=q_{m}$, condition (4.1) implies that $p_{m+1}-q_{m+1} \geq q_{m}$, so that

$$
\begin{aligned}
q_{m}^{\prime}-p_{m}^{\prime} & =q_{m}\left(\frac{1}{q_{m+1}-1}-\frac{1}{p_{m+1}-1}\right) \\
& =q_{m}\left(\frac{p_{m+1}-q_{m+1}}{\left(q_{m+1}-1\right)\left(p_{m+1}-1\right)}\right) \\
& \geq \frac{q_{m}^{2}}{\left(q_{m+1}-1\right)\left(p_{m+1}-1\right)}=q_{m}^{\prime} p_{m}^{\prime} .
\end{aligned}
$$

Thus if $p_{m}^{\prime} \geq \frac{2}{3} q_{m}^{\prime}$ we have

$$
q_{m}^{\prime}-p_{m}^{\prime} \geq \frac{2}{3}\left(q_{m}^{\prime}\right)^{2}
$$

while if $p_{m}^{\prime} \leq \frac{2}{3} q_{m}^{\prime}$ the same conclusion follows from the observation that necessarily $q_{m}^{\prime} \leq \frac{1}{2}$. Hence in any case

$$
Q_{m}-P_{m}=Q_{m-1}\left(q_{m}^{\prime}-p_{m}^{\prime}\right) \geq \frac{2}{3} Q_{m-1}\left(q_{m}^{\prime}\right)^{2}=\frac{2}{3} Q_{m} q_{m}^{\prime} .
$$

Also for each $n>m$ we have

$$
P_{n}=P_{m-1} p_{m}^{\prime} \ldots p_{n}^{\prime}=Q_{m-1} p_{m}^{\prime} \ldots p_{n}^{\prime}<Q_{m} p_{m+1}^{\prime} \ldots p_{n}^{\prime} .
$$

Because of (4.2) the numbers $p_{n}^{\prime}$ decrease with increasing $n$ and moreover $p_{m+1}^{\prime} \leq\left(p_{m}^{\prime}\right)^{2}$. Therefore we have

$$
\begin{aligned}
\sum_{n>m} P_{n} & <Q_{n} \sum_{n \geq m+1} p_{m+1}^{\prime} \ldots p_{n}^{\prime} \leq Q_{m} \sum_{n \geq 1}\left(p_{m+1}^{\prime}\right)^{n} \\
& \leq Q_{m} \sum_{n \geq 1}\left(p_{m}^{\prime}\right)^{2 n}<Q_{m} \sum_{n \geq 1}\left(q_{m}^{\prime}\right)^{2 n}=Q_{m} \frac{\left(q_{m}^{\prime}\right)^{2}}{1-\left(q_{m}^{\prime}\right)^{2}} .
\end{aligned}
$$

Since $q_{m}^{\prime} \leq \frac{1}{2}$ and the function $x \mapsto \frac{x}{1-x^{2}}$ is increasing for $0 \leq x<1$ it follows that

$$
\sum_{m>n} P_{n}<\frac{2}{3} Q_{m} q_{m}^{\prime}
$$

Combining (4.4)-(4.6) we have

$$
\theta_{\mathcal{Q}}-\theta_{\mathcal{P}}=\sum_{n \geq m}\left(Q_{n}-P_{n}\right)>Q_{m}-P_{m}>\sum_{n>m} P_{n}>0
$$

as claimed.

We can now complete the proof of Theorem III as follows. Let $g$ be a function satisfying the hypothesis of the theorem. We will show that there exists a function $G$ (depending on $g$ ) such that (1.2) fails whenever $\theta=\theta_{\mathcal{P}}$ for some $G$-good sequence $\mathcal{P}$. 
For $\mathcal{P}$ satisfying (4.1) and (4.2) we set $\theta=\theta_{\mathcal{P}}$ and write

$$
\theta_{k}=p_{1}+\sum_{1 \leq j<k} P_{j}
$$

One checks easily that for $k \geq 1$ we have

$$
\theta_{k}=p_{k} P_{k-1} \text {. }
$$

We claim that for each $k$ and each $n \leq 1 /\left(2 p_{k}^{\prime}\right)$ we have

$$
\lfloor\theta n\rfloor=\left\lfloor\theta_{k} n\right\rfloor .
$$

To see this observe that otherwise there exists some $n \leq 1 /\left(2 p_{k}^{\prime}\right)$ such that $\theta_{k} n<\lfloor\theta n\rfloor$, and then using (4.7) it follows that

$$
\theta_{k} n \leq\lfloor\theta n\rfloor-P_{k-1} \leq \theta n-P_{k-1}
$$

which implies

$$
\theta-\theta_{k} \geq n^{-1} P_{k-1} \geq 2 P_{k-1} p_{k}^{\prime}=2 P_{k} .
$$

But also we clearly have

$$
\theta-\theta_{k}=\sum_{j \geq k} P_{j}<2 P_{k}
$$

(since $P_{j+1} / P_{j}=p_{j+1}^{\prime} \leq 1 / 2$ for each $j$ ), and this contradicts (4.9). Hence (4.8) holds as claimed.

Clearly $P_{k-1}^{-1}<p_{k}$, and using (4.7) it follows that the set

$$
\left\{\lfloor\theta n\rfloor: n \leq 1 /\left(2 p_{k}^{\prime}\right)\right\}=\left\{\left\lfloor\theta_{k} n\right\rfloor: n \leq 1 /\left(2 p_{k}^{\prime}\right)\right\}
$$

can be written in the form $\left\{n: n \leq \theta /\left(2 p_{k}^{\prime}\right)\right\} \cap E$, where $E$ is the union of $P_{k-1}^{-1}$ residue classes modulo $p_{k}$. We observe that the residue class $\overline{0}$ is a subset of $E$.

For any prime $p$ we can define a function $r_{p}$ on the set of residue classes modulo $p$ by setting

$$
r_{p}(\overline{0})=\frac{2 p-1}{p^{2}}
$$

and

$$
r_{p}(\bar{h})=\frac{p-1}{p^{2}} \quad \text { if } \bar{h} \neq \overline{0}
$$

Then because $\overline{0} \subset E$ we have (using (4.7))

$$
\sum_{\bar{h} \subset E} r_{p_{k}}(\bar{h})=P_{k-1}^{-1}\left(\frac{p_{k}-1}{p_{k}^{2}}\right)+\frac{1}{p_{k}}=\theta_{k}^{-1}-\frac{\theta_{k}^{-1}}{p_{k}}+\frac{1}{p_{k}},
$$

so that 


$$
\begin{aligned}
\sum_{\bar{h} \subset E} r_{p_{k}}(\bar{h})-\theta^{-1} & \geq \sum_{\bar{h} \subset E} r_{p_{k}}(\bar{h})-\theta_{k}^{-1} \\
& \geq \frac{1-\theta_{k}^{-1}}{p_{k}} \geq \frac{1-p_{1}^{-1}}{p_{k}} \geq \frac{1}{2 p_{k}} .
\end{aligned}
$$

We can define a real-valued function $G_{0}$ on the set of primes by putting $G_{0}(p)=\max _{h} \min \left\{N \in \mathbb{N}: x>N \Rightarrow\left|T(h, p ; x)-r_{p}(\bar{h}) T(1 ; x)\right| \leq \frac{T(1 ; x)}{4 p^{2}}\right\}$

(note that Lemma 2.3 is needed to ensure that the set on the right is nonempty for each choice of $h$ and $p$ ). We can choose a function $G: \mathbb{R} \rightarrow \mathbb{R}$ satisfying

$$
G(p)>2 p G_{0}(p)
$$

for each prime $p$ and also increasing rapidly enough to ensure that

$$
g(G(x) /(2 x))>4 x
$$

for all $x \in \mathbb{R}$. Now let $\mathcal{P}$ be $G$-good and set $\theta=\theta_{\mathcal{P}}$. We write $x=1 /\left(2 p_{k}^{\prime}\right)$ and observe using (4.3) and (4.11) that then $x>G_{0}\left(p_{k}\right)$. It follows using (4.8), (4.10) and the definition of $G_{0}\left(p_{k}\right)$ that

$$
\begin{aligned}
& \left|T(\theta ; x)-\theta^{-1} T(1 ; x)\right| \\
& \quad=\left|T\left(\theta_{k} ; x\right)-\theta^{-1} T(1 ; x)\right| \\
& \quad \geq\left(\sum_{\bar{h} \subset E} r_{p_{k}}(\bar{h})-\theta^{-1}\right) T(1 ; x)-\sum_{\bar{h} \subset E}\left|T\left(h, p_{k} ; x\right)-r_{p_{k}}(\bar{h}) T(1 ; x)\right| \\
& \quad \geq\left(\frac{1}{2 p_{k}}-\frac{1}{4 p_{k}}\right) T(1 ; x)=\frac{1}{4 p_{k}} T(1 ; x) .
\end{aligned}
$$

Because $x>G\left(p_{k}\right) /\left(2 p_{k}\right)$ and $g$ is increasing, the relation (4.12) implies that $g(x)>4 p_{k}$. Hence using (4.13) we have

$$
\left|T(\theta ; x)-\theta^{-1} T(1 ; x)\right|>\frac{T(1 ; x)}{g(x)} .
$$

Thus (1.2) fails for $x=1 /\left(2 p_{k}^{\prime}\right)$ and by (4.2) such an $x$ can be chosen as large as we please. The proof is complete.

Acknowledgements. The author is indebted to his supervisor R. Nair for suggesting the use of Vaaler's polynomials, and to the SERC for financial support. 


\section{References}

[1] S. D. Chowla, Some problems of Diophantine approximation I, Math. Z. 33 (1931), $544-563$.

[2] W. J. Ellison (with M. Mendès France), Les Nombres Premiers, Hermann, 1975.

[3] S. W. Graham and G. Kolesnik, Van der Corput's Method of Exponential Sums, London Math. Soc. Lecture Note Ser. 126, Cambridge University Press, 1991.

[4] D. R. Heath-Brown, The fourth power moment of the Riemann zeta function, Proc. London Math. Soc. (3) 38 (1979), 385-422.

[5] E. Hlawka, The Theory of Uniform Distribution, AB Academic Publishers, 1984.

[6] L.-K. Hua, Introduction to Number Theory, Springer, 1982.

[7] S. Lang, Introduction to Diophantine Approximation, Addison-Wesley, 1966.

[8] H. A. Porta and K. B. Stolarsky, Wythoff pairs as semigroup invariants, Adv. in Math. 85 (1991), 69-82.

[9] S. Ramanujan, Some formulae in the analytic theory of numbers, Messenger of Math. 45 (1916), 81-84.

[10] J. D. Vaaler, Some extremal functions in Fourier analysis, Bull. Amer. Math. Soc. 12 (1985), 183-216.

DEPARTMENT OF PURE MATHEMATICS

UNIVERSITY OF LIVERPOOL

P.O. BOX 147

LIVERPOOL, L69 3BX, U.K. 